\title{
Polarimetric quantum-strong correlations with independent photons on the Poincaré sphere
}

\author{
ANDRE VATARESCU \\ Fibre-Optic Transmission of Canberra, 32 Batman Street, Canberra 2612, Australia \\ Corresponding author: andre_vatarescu@yahoo.com.au
}

\begin{abstract}
Polarization-based photonic quantum correlations can be traced back to the overlap of the polarization Stokes vectors on the Poincaré sphere between two polarization filters. Quantum-strong correlations can be obtained with independent polarization states on the Poincaré sphere. The quantum Rayleigh scattering prevents a single photon from propagating in a straight line inside a dielectric medium. The concept of quantum nonlocality is rather questionable because the quantum Rayleigh scattering in a dielectric medium destroys entangled photons.
\end{abstract}

Key words: Quantum optics; quantum correlations; polarization correlations; Stokes vectors.

\section{Introduction}

The quantum Rayleigh spontaneous and stimulated emissions were well documented four decades ago [1-2] when the first experimental results of apparently single photon propagation were incorporated in the theory of quantum optics. Even though the subject was revisited [3] to clearly find that the probability of spontaneous emission increases with the refractive index of the medium, the question of one single photon being scattered by photon-dipole interactions has been completely ignored in the professional literature of quantum optics [4]

One photon per radiation mode underpins the concept of entangled photons which, apparently, are needed to create a statistical correlation between separately measured quantum events. Yet, the quantum Rayleigh scattering prevents a single photon from propagating in a straight line inside a dielectric medium [5]; equally, inside a dielectric medium, the quantum Rayleigh stimulated emission can recapture an absorbed photon as well as coupling photons between two radiation modes, thereby creating groups of photons from individual ones [6-7].

The assumption that spontaneously emitted, parametrically down-converted individual photons cannot be amplified because of a low level of pump power would, in fact, prevent any emission in the direction of phase-matching condition because of the Rayleigh spontaneous scattering. In a nonlinear crystal pumped, e.g., with a pump wave $(p)$ and for frequency downconverted photons of $\omega_{s}+\omega_{i}=\omega_{p}$, the gain-providing medium which generates the spontaneous emission, will also amplify the initially single photons, particularly so in the direction of wavevector matching conditions, even for limited space-time overlap. A phase-pulling effect leading to $\varphi_{s}+\varphi_{i}=\varphi_{p}+\pi / 2$ also occurs [7] which is capable of countering phase-mismatch. Thus, the commonly assumed one single photon output does not physically happen. At least several photons may be associated with each individual and discrete electronic "click". A group of photons of the same frequency propagating inside a dielectric medium will follow a straight-line because a photon locally absorbed by a dipole, will be recaptured by the other photons in the group through stimulated emission.
Nevertheless, only one photon may survive the propagation to reach the photodetector.

This Letter traces the origin of the polarization-based quantum correlation function back to the overlap between the polarization Stokes vectors of the detecting filters, on the measurement Poincaré sphere. After reviewing the shortcomings of entangled states of photons in Section 2, the local measurements leading to statistical distributions of quantum correlations are specified in Section 3 by using polarization states of independent photons. By generating the polarization Stokes vectors through measurements based on Pauli spin operators, the correlation function is obtained in Section 4, with independent photons, in a manner that will reduce the complexities of operational quantum photonic systems. Physical aspects are presented in Section 5.

\section{The shortcomings of the entangled states of photons}

A recent review article [4] presents the concept of quantum nonlocality pertaining to entangled states as "the idea that measurements performed in spatially separated locations can affect each other". Such a state is given in terms of horizontal $|\mathrm{H}\rangle$ and $|\mathrm{V}\rangle$ vertical polarizations by

$$
\left|\psi_{A B}\right\rangle=\left(|\mathrm{H}\rangle_{A}|\mathrm{H}\rangle_{B}+|\mathrm{V}\rangle_{A}|\mathrm{~V}\rangle_{B}\right) / \sqrt{2}
$$

where the indexes $A$ and $B$ refer to the two entangled photons that propagate in different directions, and to be detected by spatially separated photodetectors $A$ and $B$. The state $\left|\psi_{A B}\right\rangle$ somehow remains unchanged despite the photons propagating through dielectric media of beam splitters, optical fibers, crystal polarizers, etc., and is used to calculate an ensemble correlation function between the polarization states obtained by setting the linear polarization filters to various angles with respect to a common frame of reference, i. e, $|\mathrm{H}\rangle$ and $|\mathrm{V}\rangle$. Particular values of these measurements are then added in a linear combination commonly known as the Bell-type inequality of Clauser- HorneShimony- Holt (CHSH) to infer that a quantum nonlocal effect has taken place, i.e., one measurement influencing the other.

However, this interpretation of experimental results as proof of quantum nonlocality does not stand up to physical scrutiny and has been disproved and rebutted [8-13] from various 
perspectives. The mixed quantum state $\left|\psi_{A B}\right\rangle$ of Eq. (1) is space- and time-independentand considered to be a global state which can be used in any context, anywhere, and at any time. Nevertheless, the Hilbert spaces of the two photons move away from each other and do not spatially overlap, so that any composite Hilbert space is mathematically generated by means of a tensor product. Even so, the absence of a Hamiltonian of interaction renders any suggestion of a mutual influence rather questionable.

The quantum evaluation of the correlation function is carried out with two polarization filter operators $\hat{\sigma}_{A}$ and $\hat{\sigma}_{B}$ and given by:

$$
\begin{aligned}
& E_{C}\left(\theta_{A} ; \theta_{B}\right)=\left\langle\psi_{A B}\left|\hat{\sigma}_{A} \otimes \hat{\sigma}_{B}\right| \psi_{A B}\right\rangle= \\
& =\left[{ }_{A}\left\langle\mathrm{H}\left|\hat{\sigma}_{A}\right| \mathrm{H}\right\rangle_{A B}\left\langle\mathrm{H}\left|\hat{\sigma}_{B}\right| \mathrm{H}\right\rangle_{B}+{ }_{A}\left\langle\mathrm{~V}\left|\hat{\sigma}_{A}\right| \mathrm{V}\right\rangle_{A B}\left\langle\mathrm{~V}\left|\hat{\sigma}_{B}\right| V\right\rangle_{B}+\right. \\
& \left.+{ }_{A}\left\langle\mathrm{H}\left|\hat{\sigma}_{A}\right| \mathrm{V}\right\rangle_{A B}\left\langle\mathrm{H}\left|\hat{\sigma}_{B}\right| \mathrm{V}\right\rangle_{B}+{ }_{A}\left\langle\mathrm{~V}\left|\hat{\sigma}_{A}\right| \mathrm{H}\right\rangle_{A B}\left\langle\mathrm{~V}\left|\hat{\sigma}_{B}\right| \mathrm{H}\right\rangle_{B}\right] / 2= \\
& =\cos 2\left(\theta_{A}-\theta_{B}\right)
\end{aligned}
$$

where the Pauli spin operators of the polarizations are denoted $\hat{\sigma}_{k}=\sin \left(2 \theta_{k}\right) \hat{\sigma}_{1}+\cos \left(2 \theta_{k}\right) \hat{\sigma}_{3}$ with $k=A$ or $B$, the angle $\theta_{k}$ specifies the rotation of a linear polarization filter and the projecting Pauli operators are in this case

$\hat{\sigma}_{1}=|\mathrm{H}\rangle\langle\mathrm{V}|+| \mathrm{V}\rangle\langle\mathrm{H}|$ and $\hat{\sigma}_{3}=|\mathrm{H}\rangle\langle\mathrm{H}|-| \mathrm{V}\rangle\langle\mathrm{V}|$. The first operator corresponds to an interference effect of the two projected measuring eigenstates and requires for a non-zero value that the two eigenmodes be simultaneously populated, that is, more than one photon should reach the same polarization analyser. Additionally, the correlation function $E_{c}\left(\theta_{A} ; \theta_{B}\right)$ contains on its third line of Eq. (2) matrix elements which involve non-coincident photons because for the states of Eq. (1) the same state of polarization occurs for each single event or measurement of the statistical ensemble, namely, either $|\mathrm{H}\rangle_{A}$ and $|\mathrm{H}\rangle_{B}$, or $|\mathrm{V}\rangle_{A}$ and $|\mathrm{V}\rangle_{B}$ are present simultaneously. If a collapse of the wave function takes place upon detection of a photon at either location, then the two separate measurements do not coincide as required by Eq. (2). In this case, a local measurement vanishes, e.g., $\left\langle\psi_{A B}\left|\hat{\sigma}_{A} \otimes \hat{I}_{B}\right| \psi_{A B}\right\rangle=0$, which leads to a physical contradiction as local experimental outcomes determine the state of polarization to be compared with its pair quantum state.

Photons polarized parallel to the reference coordinates will pass randomly through polarization filters or analysers as a result of quantum Rayleigh scatterings of photons [1-3], [10]. These photons will emerge with the same state of polarization as those of the filters, and the corresponding Stokes parameters of the polarization state vector $\vec{S}$ on the Poincaré sphere are calculated as the expectation value of the Pauli spin vector operator $\hat{\sigma}=\left(\hat{\sigma}_{1}, \hat{\sigma}_{2}, \hat{\sigma}_{3}\right)$ for the Jones vectors [14] $\left|u\left(\theta_{k}\right)\right\rangle=\cos \theta_{k}|\mathrm{H}\rangle+\sin \theta_{k}|\mathrm{~V}\rangle$ of the filters, that is :

$$
\vec{s}_{k}=\left\langle u\left(\theta_{k}\right)|\hat{\sigma}| u\left(\theta_{k}\right)\right\rangle
$$

This relation connecting the correlation overlap between two polarization state vectors $\left|u_{k}\right\rangle=\left|u\left(\theta_{k}\right)\right\rangle$ in the Jones representation and the overlap or correlation of their corresponding Stokes vectors $\vec{S}_{k}$ on the Poincaré sphere is given [14] by:

$$
\begin{gathered}
\left|\left\langle u_{A} \mid u_{B}\right\rangle\right|^{2}=\frac{1}{2}\left(1+\vec{s}_{A} \cdot \vec{s}_{B}\right) \\
E_{c}\left(\theta_{A} ; \theta_{B}\right)=\vec{s}_{A} \cdot \vec{s}_{B}=\cos 2\left(\theta_{A}-\theta_{B}\right)
\end{gathered}
$$

to obtain in Eq. (4b) the same correlation function as in Eq. (2);; In the next Section, independent states of photons will be identified in the Jones representation of polarization states and the overlap of the corresponding Stokes vectors on the Poincaré sphere will explain the quantum correlation as the mathematical result of the two polarization filters projecting onto each other in the joint Hilbert space of the measurements.

It is claimed that the presence of two operators in the correlation tensor product results in stronger correlation values for entangled states. Yet, the spatially separate measurements of one photon reaching each polarization filter can be identified specifically by using the identity operators $\hat{I}_{k}$ for each polarization filter, that is:

$$
\begin{aligned}
E_{C}\left(\theta_{A} ; \theta_{B}\right) & =\left(\left\langle\psi_{A B}\right| \hat{\sigma}_{A} \otimes \hat{I}_{B}\right)\left(\hat{I}_{A} \otimes \hat{\sigma}_{B}\left|\psi_{A B}\right\rangle\right) \\
& =\left\langle\Phi_{A} \mid \Phi_{B}\right\rangle
\end{aligned}
$$

In this way, the correlation function is associated with the overlap, or fidelity, of two state vectors $\left|\Phi_{k}\right\rangle$ displayed on the Poincaré sphere as an inner product. As a consequence, the same correlation function between the polarization filters of the detectors can be associated with independent states of photons as presented in the next Section.

\section{Quantum correlations of independent photons}

The correlation function $E_{C}$ for the detection of two photons $A$ and $B$ of the state $\left|\psi_{A B}\right\rangle$ of Eq. (1) is defined as the sum of averaged products of any two eigenvalues +1 or -1 assigned to eigenstates $|x(\theta)\rangle$ and $|y(\theta)\rangle$, respectively, involving the probabilities of their coincident detections, i.e. $P_{++} ; P_{--} ; P_{-+} ; P_{+-}$for various settings $\theta_{A}$ and $\theta_{B}$ of the polarization filters:

$$
\begin{aligned}
E_{c}\left(\theta_{A} ; \theta_{B}\right) \equiv & P_{++}\left(\theta_{A} ; \theta_{B}\right)+P_{--}\left(\theta_{A} ; \theta_{B}\right)- \\
& -P_{+-}\left(\theta_{A} ; \theta_{B}\right)-P_{-+}\left(\theta_{A} ; \theta_{B}\right)
\end{aligned}
$$

The probabilities are linked experimentally to the counts $N_{i, j}$ of coincident photons through the equality $N_{i, j}\left(\theta_{A} ; \theta_{B}\right)=$ $P_{i j}\left(\theta_{A} ; \theta_{B}\right) N_{t o t}$, where $i, j=+;-$ and $N_{\text {tot }}$ is the total number of coincident photons. In the case of independent statistical events at the two spatially separated detectors, the joint probability becomes the product of the independent probabilities, that is $P_{i j}\left(\theta_{A} ; \theta_{B}\right)=P_{i}\left(\theta_{A}\right) P_{j}\left(\theta_{B}\right)$. As a simple example, let us consider the detecting filter's polarization eigenstates, for $k=A$; $B$, in the reference frame of coordinates which lies in the measurement Hilbert space, common to the two locations, that is:

$$
\begin{gathered}
\left|x\left(\theta_{k}\right)\right\rangle=\cos \theta_{k}|x\rangle+\sin \theta_{k}|y\rangle \\
\left|y\left(\theta_{k}\right)\right\rangle=-\sin \theta_{k}|x\rangle+\cos \theta_{k}|y\rangle
\end{gathered}
$$


rotated from the reference states $|x\rangle$ and $|y\rangle$ by an angle $\theta_{k}$.

For the same state of linear polarization rotated by an angle $\varphi$ from the reference coordinates, that is

$$
|\Psi(\varphi)\rangle=\cos \varphi|x\rangle+\sin \varphi|y\rangle
$$

reaching both detectors, the correlation function is derived in the remainder of this Section. The case of different input rotation angles will be derived in Section 4.

The equality of Eq. (6) can be rewritten, for independent statistics, as:

$$
\begin{aligned}
E_{c}\left(\theta_{A} ; \theta_{B}\right) & =\left[P_{+}\left(\theta_{A}\right)-P_{-}\left(\theta_{A}\right)\right]\left[P_{+}\left(\theta_{B}\right)-P_{-}\left(\theta_{B}\right)\right]= \\
& =P\left(\theta_{A}\right) \otimes P\left(\theta_{B}\right)
\end{aligned}
$$

With the vectorial structure of $P\left(\theta_{k}\right)=\left(P_{+}\left(\theta_{k}\right) ;-P_{-}\left(\theta_{k}\right)\right)$, the dyadic or tensor product is a shorthand notation for the direct product of the two $P\left(\theta_{k}\right)$ vectors. The tensor product is commonly used in quantum mechanics to point out that two different Hilbert spaces may have different systems of coordinates. Otherwise, the direct product is more practical.

As we can see from this equality (9), the correlation functions can be obtained with any type of probabilities and it will be further developed below to include independent photons reaching identical detecting configurations.

From Eqs. (7) the projection operators for the two measuring eigenstates are

$$
\begin{aligned}
& \hat{P}_{+}(\theta)=|x(\theta)\rangle\langle x(\theta)| \\
& \hat{P}_{-}(\theta)=|y(\theta)\rangle\langle y(\theta)|
\end{aligned}
$$

The polarization observable $\hat{\sigma}\left(\theta_{k}\right)$ in Eq. (9) has the form:

$$
\hat{\sigma}\left(\theta_{k}\right)=\hat{P}_{+}\left(\theta_{k}\right)-\hat{P}_{-}\left(\theta_{k}\right)=\sin \left(2 \theta_{k}\right) \hat{\sigma}_{1}+\cos \left(2 \theta_{k}\right) \hat{\sigma}_{3}
$$

the projecting Pauli operators being $\hat{\sigma}_{1}=|x\rangle\langle y|+| y\rangle\langle x|$ and $\hat{\sigma}_{3}=|x\rangle\langle x|-| y\rangle\langle y|$. The angle $\theta_{k}$ of a rotated polarization filter is set in the Jones representation relative to a measurement basis of reference or generic eigenstates in the Hilbert space of $\mathcal{H}=\mathcal{H}_{A} \otimes \mathcal{H}_{B}$.

By combining Eqs. (8), (9) and (11), the correlation function is evaluated for $P_{+}\left(\theta_{A}\right)-P_{-}\left(\theta_{A}\right)=\left\langle\hat{\sigma}\left(\theta_{k}\right)\right\rangle$ to be:

$$
\begin{aligned}
E_{c}\left(\theta_{A} ; \theta_{B}\right) & =\left\langle\Psi\left|\hat{\sigma}\left(\theta_{A}\right)\right| \Psi\right\rangle\left\langle\Psi\left|\hat{\sigma}\left(\theta_{A}\right)\right| \Psi\right\rangle= \\
& =\cos 2\left(\theta_{A}-\varphi\right) \cos 2\left(\theta_{B}-\varphi\right)
\end{aligned}
$$

The correlation function for $\theta_{A}=0, \theta_{B}=\theta$ and $\varphi=$ 0 or $\pi / 2$ in Eq. (12) becomes $E_{c}(0 ; \theta)=\cos 2 \theta$, which is the result for entangled states of photons [9], [15]. With only one state of polarization being populated in Eq. (8), this example points to the correlation between the polarization analysers as the source of experimentally detected correlations, as opposed to an assumed quantum nonlocality.

The correlation function is a numerical calculation as opposed to a physical interaction. Thus, the numerical comparison of the data sets is carried out at a third location $C$ where the reference system of coordinates is located for comparison or correlation calculations of the two sets of measured data, and does not require physical overlap of the observables whose operators are aligned with the system of coordinates of the measurement Hilbert space onto which the detected state vectors are mapped. In this case, the correlation operator $\hat{C}=\hat{\sigma}_{A} \otimes \hat{\sigma}_{B}$ of Eq. (2) can be reduced to [14; Eq. (A6)]:

$$
\hat{C}=(\boldsymbol{a} \cdot \hat{\sigma})(\boldsymbol{b} \cdot \hat{\sigma})=\boldsymbol{a} \cdot \boldsymbol{b} \hat{I}+i(\boldsymbol{a} \times \boldsymbol{b}) \cdot \hat{\sigma}
$$

where the polarization vectors $\boldsymbol{a}$ and $\boldsymbol{b}$ identify the orientation of the detecting polarization filters in the Stokes representation, and $\hat{\sigma}=\left(\hat{\sigma}_{1}, \hat{\sigma}_{2}, \hat{\sigma}_{3}\right)$ is the Pauli spin vector (with $\hat{\sigma}_{2}=$ $i \hat{\sigma}_{1} \hat{\sigma}_{3}$ ). The presence of the identity operator in Eq. (13) implies that, when the last term vanishes for a linear polarization state, the correlation function is determined by the orientations of the polarization filters. This can be easily done with independent and linearly polarized states of Eq. (8), because of a zero-expectation value for $\hat{\sigma}_{2}$, namely $\left\langle\Psi\left|\hat{\sigma}_{2}\right| \Psi\right\rangle$ $=0$, which implies that the commutator relation $\left\langle\Psi\left|\left[\hat{\sigma}_{3}, \hat{\sigma}_{1}\right]\right| \Psi\right\rangle=0$ also vanishes for the state of Eq. (8).

\section{Quantum correlations with independent photons on the Poincaré sphere.}

In order to emphasize the role played by independent states of photons, these states $|\psi\rangle_{k}$ will be expanded in terms of the polarization eigenstates of the reference system of coordinates that will also define the joint Poincaré sphere. The states are, with $k=A$ or $B$ :

$$
\left|\psi_{k}\right\rangle=\cos \varphi_{k}|x\rangle+\sin \varphi_{k}|y\rangle
$$

for two different angles $\varphi_{A}$ and $\varphi_{B}$, relative to the $x$-axis of reference in the measurement-related Hilbert space onto which the detected states are projected by detectors $A$ and $B$, respectively.

The polarization operator $\hat{\sigma}$ projects the incoming states onto the measurement Hilbert space for comparison of the two separate data sets. The polarization measurement operators of Eq. (11) produce the output states

$$
\left|\Phi_{k}\right\rangle=\sin \left(2 \theta_{k}\right) \hat{\sigma}_{1}\left|\psi_{k}\right\rangle+\cos \left(2 \theta_{k}\right) \hat{\sigma}_{3}\left|\psi_{k}\right\rangle
$$

which analogously to the overlapping inner product of the last line of Eq. (5), lead to the correlation function of

$$
E_{c}=\left\langle\Phi_{A} \mid \Phi_{B}\right\rangle=\cos \left[2\left(\theta_{A}-\theta_{B}\right)-\left(\varphi_{A}-\varphi_{B}\right)\right]
$$

Recalling that the phases $\varphi_{k}$ are set in the Jones representation, this result is consistent with Eq. (4) linking the overlap of the Jones vectors to the correlation of the corresponding Stokes vectors on the Poincaré sphere where the angle $2 \varphi_{k}$ applies, that is:

$$
\begin{array}{r}
E_{c}=\vec{s}_{1} \cdot \vec{s}_{2}=2 \cos ^{2}(\Delta \phi)-1=\cos 2(\Delta \phi) \\
\Delta \phi=\theta_{A}-\theta_{B}-\left(\varphi_{A}-\varphi_{B}\right)
\end{array}
$$


The quantum correlation function of Eq. (17) between two independent states of polarized photons is equivalent to the overlap of their Stokes vectors on the joint Poincaré sphere of the measurement Hilbert space. Quantum-strong correlation are possible with independent states of photons because the source of the correlation is the polarization states of the detecting filters or analysers, making any claim of quantum nonlocality unnecessary.

\section{Physical aspects of simultaneous measurements of independent photons}

Since the same correlation functions are derived for independent and single qubits generated through quantum Rayleigh conversion of photons - from initially entangled polarized photons - as for the initially entangled photons, it follows that the violations of any type of relevant Bell inequalities will also take place in the same way. Yet, the correlations result from similar, if not identical, distributions of polarization states as opposed to what is conceptually believed to be a non-local quantum effect which has an unspecified nature but is being pursued because of historical reasons.

Once the same correlation functions are derived using only states of polarizations emitted spontaneously by the quantum Rayleigh conversion of photons, no other physical processes are required to explain the experimental results.

Let us now consider a few characteristics associated with local realism [16] of quantum measurements in the context of quantum Rayleigh conversion of photons:

1. Locality of measurements is supported by the use of single and independent photonic qubits to explain the experimental results of apparently enhanced correlations of outcomes.

2. Randomness of experimental parameters stems from the quantum Rayleigh spontaneous emission that generates the projection from the polarization state $|x\rangle$ of the input photons to the rotated polarization state $\left|\psi_{k}\right\rangle=\cos \varphi_{k}|x\rangle+$ $\sin \varphi_{k}|y\rangle$ of Eq. (14).

3. Realism of values carried by the detected photons is indicated by the physical effect of the measuring operators on the detected photons in quantum states of Eq. (14). As the expectation values of the product operator $\left\langle\hat{\sigma}_{1} \hat{\sigma}_{3}\right\rangle$ are found to vanish for the pure states of Eq. (14) projected onto the measurement Hilbert space, i.e. $\quad\left\langle\psi(\varphi)\left|\hat{\sigma}_{3} \hat{\sigma}_{1}\right| \psi(\varphi)\right\rangle=$ $=\left\langle\psi(\varphi)\left|\hat{\sigma}_{1} \hat{\sigma}_{3}\right| \psi(\varphi)\right\rangle=0$ as $\hat{\sigma}_{1} \hat{\sigma}_{3}\left|\psi_{k}\right\rangle=\left|\psi_{k}(\varphi+\pi / 2)\right\rangle$, each term of the resulting commutative relation vanishes and we obtain $\left\langle\psi_{k}\left|\left[\hat{\sigma}_{1}, \hat{\sigma}_{3}\right]\right| \psi_{k}\right\rangle=0$ for the lower limit of the Heisenberg uncertainty relation which needs to be evaluated in the context of a set of wave functions. Thus, the output value is indicative of the input one, and each term of the commutator vanishes for the wave functions $\left|\psi_{k}(\varphi)\right\rangle$ of Eq. (14). Consequently, the simultaneous measurement of these two operators in the context of the single and independent qubit wave functions is capable of identifying the incoming state as well as the measured one. Thus, a physically meaningful identification of wavefunctions will enable simultaneous measurements of well-defined values.

\section{Conclusions}

The analysis presented in this Letter was motivated by physically meaningful interactions of quantum Rayleigh conversion of photons which scatter entangled photons. Quantum-strong correlations of polarized photons can be obtained with independent inputs to identical measuring devices and configurations. The correlation function is reminiscent of the overlap between two polarization Stokes vectors on the Poincaré sphere which can be derived from the Jones vectors.

The analysis supports reference [9] in its statement that "There is no mystery. There is no quantum nonlocality". It is the physical process that gives rise to a wave function. The opposite approach of relying on mathematical complexities to conjure up physical processes is bound to generate "'quantum mysteries". This physical approach is developed in reference [17].

\section{References}

1. W. H. Louisell, Quantum Statistical Properties of Radiation, John Wiley \& Sons, 1973.

2. D. Marcuse, Principles of Quantum Electronics, Academic Press, 1980.

3. R. J. Glauber and M. Lewenstein, "Quantum optics of dielectric media", Phys. Rev. A, 43, 467 (1991).

4. D. Paneru, E. Cohen, R. Fickler, R. W. Boyd and E. Karimi, "Entanglement: quantum or classical? “, Rep. Prog. Phys., 83, 064001 (2020).

5. A. Vatarescu, "Photonic coupling between quadrature states of light in a homogeneous and optically linear dielectric medium", J. Opt. Soc. Am. B, 31, 1741-1745, (2014).

6. A. Vatarescu, "Phase-Sensitive Amplification with Low Pump Power for Integrated Photonics ", OSA Advanced Photonics Congress, paper ID: IM3A.6., 2016.

7. A. Vatarescu, "Photonic Quantum Noise Reduction with Low-Pump Parametric Amplifiers for Photonic Integrated Circuits", Photonics 3, 61, (2016).

8. Robert B. Griffiths, "Nonlocality claims are inconsistent with Hilbertspace quantum mechanics", Phys. Rev. A 101, 022117 (2020)..

9. F. J. Tipler, "Quantum nonlocality does not exist," PNAS 111 (31), 11281-11286, (2014). doi.org/10.1073/pnas.1324238111

10. A. Vatarescu, "The Scattering and Disappearance of Entangled Photons in a Homogeneous Dielectric Medium," Rochester Conference on Coherence and Quantum Optics (CQO-11), (2019). doi.org/10.1364/CQO.2019.M5A.19.

11. S. Boughn, "Making Sense of Bell's Theorem and Quantum Nonlocality", Found. Phys., 47, 640-657 (2017).

12. A. Khrennikov, "Get Rid of Nonlocality from Quantum Physics", Entropy, 21, 806 (2019).

13. M. Kupczynski, "Closing the Door on Quantum Nonlocality", Entropy, 20, 877 (2018).

14. J.P. Gordon and H. Kogelnik, "PMD fundamentals: Polarization mode dispersion in optical fibers", PNAS, 97 (9), 4541-4550, (2000) www.pnas.org/cgi/content/abstract/97/9/4541.

15. J. C. Garrison and R.Y. Chiao, Quantum Optics, Oxford University Press, 2008.

16. D. J. Griffiths, 2005, Introduction to Quantum Mechanics, Publisher: Pearson Prentice Hall.

17. A. Vatarescu, The Physical Reality of Applied Quantum Optics, 2021, Cambridge Scholars Publishing, UK. 